\title{
Simulation of the Raman Spectroscopy of Multi-Layered Carbon Nanomaterials
}

\author{
Pritesh M. Tailor, Richard J. Wheatley and Nicholas A. Besley* \\ School of Chemistry, University of Nottingham, University Park, Nottingham, NG7 2RD, \\ $U K$. \\ E-mail: nick.besley@nottingham.ac.uk
}

\begin{abstract}
Multi-layered carbon nanomaterials can have an important role in modern nanotechnology. Raman spectroscopy is a widely used analytical technique that is used to characterise the structure of these materials. In this work, an approach based upon an empirical potential for the simulation of the Raman spectroscopy of carbon nanomaterials [Carbon, 113 (2017) 299] is extended through the addition of a term to describe the Van der Waals interaction between layers of $\mathrm{sp}^{2}$ hybridised carbons. The resulting model accurately describes the properties of the shearing modes of few layer graphene and is used to characterise the low frequency modes of multi-walled carbon nanotubes and carbon nanofibres.
\end{abstract}




\section{Table of Contents Graphic}

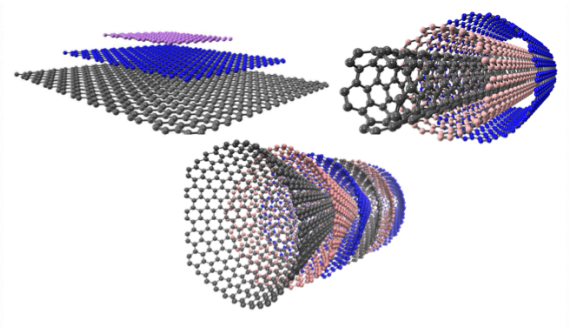

An empirical potential based model for simulating the Raman spectroscopy of layered carbon nanomaterials is introduced. 


\section{Introduction}

Carbon nanomaterials, such as carbon nanotubes $(\mathrm{CNTs})^{1}$ and graphene, ${ }^{2}$ can have remarkable structural, mechanical, magnetic and electronic properties, and these properties can be exploited in modern nanotechnology. ${ }^{3,4}$ Carbon nanomaterials that contain several layers, for example multi-walled carbon nanotubes or bi-layer graphene, add a further dimension to carbon nanoscience. These materials can exhibit different properties compared with their single layer counterparts with lower aspect ratios, as well as bypassing limitations in the processing and assembly of single layer systems. ${ }^{3}$

The high level of interest in the processing, modification, and customisation of these materials has led to a demand for techniques that can accurately characterise carbon nanomaterials. Raman spectroscopy is a fast and non-destructive technique that is well suited to characterise key elements of these materials, and is routinely used to provide information on the structure, bonding and environment of $\mathrm{CNTs}^{5}$ and graphene, ${ }^{6}$ including details on CNT diameter, chirality and defects. The most intense mode in the Raman spectroscopy of graphene and carbon nanotubes is the $\mathrm{G}$ band which arises from planar vibrations of the carbon atoms. For CNTs this band can be split into the $\mathrm{G}^{+}$and $\mathrm{G}^{-}$bands, which correspond to in-plane movements along and perpendicular to the CNT axis, respectively. The $\mathrm{G}$ band can be used to determine whether a CNT is metallic or semi-conducting, and allows for the qualitative assignment of the chiral indices of a CNT. A further key mode in the Raman spectroscopy of CNTs is the radial breathing mode (RBM) which corresponds to a coherent expansion and contraction of the carbon atoms in the radial direction and is known to depend on the diameter of the CNT. A further weak band is the disorder-induced D band, which is associated with $s p^{3}$ defects in CNTs, ${ }^{7}$ and the ratio of the intensities of the $\mathrm{G}$ and D bands can be used to probe the degree of covalent functionalization of CNTs. ${ }^{8}$

Recent reviews summarise the key experimental measurements on the Raman spectroscopy of few-layer graphene (FLG). ${ }^{9,10}$ Ferrari and co-workers ${ }^{11}$ have reported Raman measurements of FLG of up to 10-layers revealing the Raman active interlayer shear mode 
which involves the relative motion of adjacent planes of carbon as illustrated in figure 1. The bulk shear mode of graphite lies between 43 and $44 \mathrm{~cm}^{-1}$, and it was shown that the highest frequency Raman active interlayer shear mode has a frequency that is dependent on the number of layers $\left(N_{L}\right)$ according to

$$
\omega_{N}=\frac{1}{\sqrt{2} \pi c} \sqrt{\frac{\alpha}{\mu}} \sqrt{1+\cos \left(\frac{\pi}{N_{L}}\right)}
$$

where $\mu$ is the mass per unit area of single layer graphene and has a value of $7.6 \times 10^{-27}$ $\operatorname{kg} \AA^{-2}$ and $c$ is the speed of light. Through fitting to experimental data the parameter $\alpha$ which represents the interlayer coupling strength was estimated to be $12.8 \times 10^{18} \mathrm{Nm}^{-1}$. It was noted that this shear mode will be present in all layered materials and can be used to probe interlayer interactions. In contrast to the shearing mode, the frequency of the $\mathrm{G}$ band was not dependent on $N_{L}$. The Raman spectra for the shearing modes was reported to be dependent on the stacking pattern in tri-layer graphene. ${ }^{12}$ Lui and Heinz have studied the evolution of the layer breathing modes of FLG using electronically resonant overtone Raman bands. $^{13}$
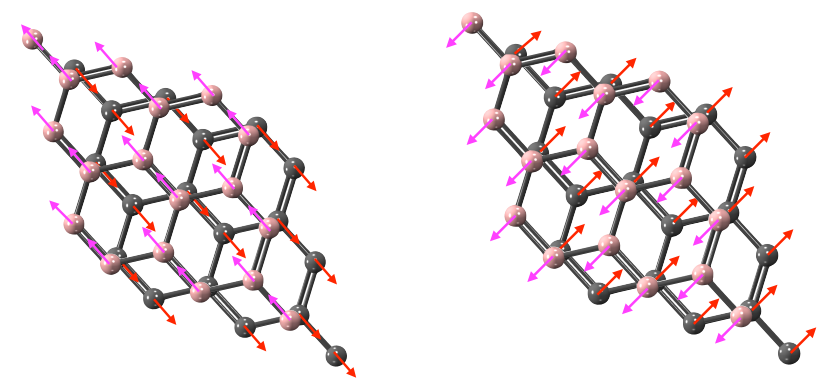

Figure 1: The normal mode displacement of the doubly degnerate $\mathrm{E}_{2 \mathrm{~g}}$ shear mode $\left(31 \mathrm{~cm}^{-1}\right)$ in bilayer graphene.

A number of studies have measured the Raman spectroscopy of multi-walled carbon nanotubes (MWCNTs) which reveal low frequency Raman modes which are associated with RBMs. ${ }^{14-16}$ These modes are of interest owing to their dependence on the diameters of the concentric tubes, and a relationship between the RBM frequency and the inner-diameter of a 
double-walled carbon nanotube has been developed. ${ }^{17}$ However, the situation for MWCNTs is more complex since the RBMs of the concentric tubes will not act as independent oscillators because the Van der Waals interaction between the tubes can be sufficiently large that the individual vibrations couple together. The interpretation of the Raman spectra of MWCNTs is more complex than for single-walled nanotubes, and a lack of theoretical studies means that the interpretation of the experimental spectra is usually based on established results obtained for single-walled tubes. ${ }^{18}$

Accurate simulations of the Raman spectroscopy of carbon nanomaterials have the potential to assist with the interpretation of experimental measurements since they can establish the relationship between the structure and the observed Raman features. This is particularly important for materials with structural defects or disorder. ${ }^{19}$ A number of groups have studied the infrared and Raman spectroscopy of carbon nanomaterials based upon density functional theory (DFT) based harmonic frequency calculations. These studies include $\mathrm{C}_{60}$ and $\mathrm{C}_{70},{ }^{20-25}[\mathrm{n}]$ cycloparaphenylenes ${ }^{26,27}$ and a nanotorus. ${ }^{28}$ Performing a DFT based harmonic frequency analysis for CNTs is challenging owing to the computational cost. In general, two approaches to the study of nanotubes are adopted. In the first of these approaches, a finite model of the nanotube is used, which can be capped using a fullerene fragment or with hydrogen atoms. ${ }^{29,30}$ Examples of this approach include calculations of the diameter dependence of the RBM of zigzag nanotubes, ${ }^{31}$ studies of vibrational spectroscopy of ultra-small nanotubes ${ }^{32}$ and larger nanotubes consisting of up to 120 carbon atoms. ${ }^{33}$ The Raman spectroscopy of larger nanotubes have been studied exploiting a cartesian coordinate transfer technique. ${ }^{34}$ Alternatively, a periodic formalism can be adopted and the effect of SW and di-vacancy defects on Raman spectra has been studied ${ }^{35}$ using plane-wave based periodic DFT.

Other computationally less expensive methods including tight-binding DFT have been applied to study the vibrational spectroscopy of fullerenes ${ }^{36,37}$ and CNTs. ${ }^{38,39}$ The vibrational density of states of carbon nanotubes have been simulated based upon a semi-empirical 
potential. ${ }^{40,41}$ Recently, we introduced an empirical model for the simulation of the Raman spectroscopy of carbon nanomaterials in which the Murrell-Mottram potential was reparameterised to reproduce the DFT structure and Hessian matrix of $\mathrm{C}_{60} \cdot{ }^{42}$ The harmonic frequencies calculated using this potential are combined with Raman intensities computed using the bond polarisation model $(\mathrm{BPM})^{43}$ and allowed the Raman spectra of carbon materials consisting of several thousand carbon atoms to be simulated. Subsequently, the role of defects on the Raman spectroscopy of CNTs and graphene, and the Raman spectroscopy of carbon nanotube junctions were studied.

The shearing and breathing modes of FLG have been studied using a generalized force constant model. ${ }^{44}$ An inter-layer interaction term has been included in a tight-binding DFT approach and used to study the low frequency phonons of FLG, MWCNTs and carbon onions. ${ }^{45}$ The RBMs of MWCNTs have been studied using a range of approaches including treating the nanotubes as a set of concentric cylindrical shells with an inter-tube interaction based upon the Lennard-Jones potential, ${ }^{46}$ addition of a Lennard-Jones Van der Waals term to an intra-layer force constant model $^{47}$ and valence force field model. ${ }^{48}$ In this paper we describe the extension of our Murrell-Mottram based potential to treat multi-layered carbon materials by the addition of a dispersion term. This term is adapted from the dispersion correction of Grimme ${ }^{49}$ that is routinely included in DFT calculations. Raman spectra are computed using the BPM, and subsequently the low frequency Raman active modes of FLG, MWCNTs and nanofibres are studied.

\section{Computational Details}

The Murrell-Mottram (MM) potential ${ }^{50}$ represents the interaction between atoms through a sum of two-body and three-body contributions. For a system of $N$ atoms, the potential 
takes the following form:

$$
E=\sum_{i}^{N} \sum_{j=i+1}^{N} V_{i j}^{(2)}+\sum_{i}^{N} \sum_{j=i+1}^{N} \sum_{k=j+1}^{N} V_{i j k}^{(3)}
$$

where

$$
\begin{aligned}
V_{i j}^{(2)} & =-D\left(1+a_{2} \rho_{i j}\right) \exp \left(-a_{2} \rho_{i j}\right) \\
V_{i j k}^{(3)} & =D P\left(Q_{1}, Q_{2}, Q_{3}\right) \exp \left(-a_{3} Q_{1}\right) \\
\rho_{i j} & =\left(r_{i j}-r_{e}\right) / r_{e} .
\end{aligned}
$$

The two-body potential $V_{i j}^{(2)}$ is represented by a Rydberg function, where $r_{i j}$ is the distance between atoms $i$ and $j$. $D$ and $r_{e}$ are parameters that are chosen such that the energy and structure are described accurately. The terms $\exp \left(-a_{2} \rho_{i j}\right)$ and $\exp \left(-a_{3} Q_{1}\right)$ are damping functions which depend on the parameters $a_{2}$ and $a_{3}$, and they ensure the potential converges to zero energy at infinite interatomic separation. $P\left(Q_{1}, Q_{2}, Q_{3}\right)$ is a quartic polynomial

$$
\begin{aligned}
P\left(Q_{1}, Q_{2}, Q_{3}\right) & =c_{0}+c_{1} Q_{1}+c_{2} Q_{1}^{2}+c_{3}\left(Q_{2}^{2}+Q_{3}^{2}\right) \\
& +c_{4} Q_{1}^{3}+c_{5} Q_{1}\left(Q_{2}^{2}+Q_{3}^{2}\right) \\
& +c_{6}\left(Q_{3}^{3}-3 Q_{3} Q_{2}^{2}\right)+c_{7} Q_{1}^{4}+c_{8} Q_{1}^{2}\left(Q_{2}^{2}+Q_{3}^{2}\right) \\
& +c_{9}\left(Q_{2}^{2}+Q_{3}^{2}\right)^{2}+c_{10} Q_{1}\left(Q_{3}^{3}-3 Q_{3} Q_{2}^{2}\right)
\end{aligned}
$$

where $Q_{i}$ are symmetrical coordinates

$$
\begin{aligned}
Q_{1} & =\frac{1}{\sqrt{3}}\left(\rho_{i j}+\rho_{i k}+\rho_{j k}\right) \\
Q_{2} & =\frac{1}{\sqrt{2}}\left(\rho_{i j}+\rho_{i k}\right) \\
Q_{3} & =\frac{1}{\sqrt{6}}\left(2 \rho_{i j}-\rho_{i k}-\rho_{j k}\right) .
\end{aligned}
$$


The term $Q_{1}$ describes the perimeter of the interaction triangles, while $Q_{2}$ and $Q_{3}$ describe the distortions of the triangles from being equilateral and $c_{0}$ to $c_{10}$ are parameters that need to be determined.

Parameters for the MM potential have been developed for a wide range of elements for the study of metal clusters, ${ }^{51-58}$ and also for non-metallic elements including carbon, silicon and germanium. ${ }^{58-60}$ In an earlier parameterisation for carbon reported by Eggen et al. ${ }^{60}$ the potential was fitted to the phonon frequencies and elastic constants of diamond and to the cohesive energy and intralayer spacing of graphite in addition to other structural data. It was noted that small carbon clusters and fullerenes will both have $\pi$-bonding electronic effects, which are missing in diamond which has only $s p^{3}$ carbons. Consequently, the potential will not be well suited to describe CNTs and graphene-based materials. Recently, we reparameterised the MM potential to describe the structure and vibrational frequencies of carbon nanomaterials using a Monte-Carlo hessian-matching approach to reproduce data from DFT calculations. ${ }^{42}$ This potential was applied to study the vibrational spectroscopy of single-walled carbon nanotubes and graphene.

In order to model multi-layer carbon materials, the potential needs to be extended to include the Van der Waals interactions between the layers. A dispersion term has been added to the MM potential, so the potential now has the form

$$
E=\sum_{i}^{N} \sum_{j=i+1}^{N} V_{i j}^{(2)}+\sum_{i}^{N} \sum_{j=i+1}^{N} \sum_{k=j+1}^{N} V_{i j k}^{(3)}+\sum_{i}^{N} \sum_{j=i+1}^{N} V_{i j}^{(D i s p)} .
$$

The dispersion term introduced by Grimme ${ }^{49}$ for including dispersion interactions into DFT calculations is used.

$$
V_{i j}^{(D i s p)}=-s_{6} \sum_{i}^{N-1} \sum_{j=i+1}^{N} \frac{C_{6}^{i j}}{R_{i j}^{6}} f_{d m p}\left(R_{i j}\right)
$$

where $C_{6}^{i j}$ is the dispersion coefficient for atom pair $i j, s_{6}$ is a global scaling factor to account for the different behaviour of the intermolecular potential especially at intermediate distances, and $R_{i j}$ is the inter-atomic distance between atoms $i j$. The damping function 
$f_{d m p}\left(R_{i j}\right)$ is given by

$$
f_{d m p}\left(R_{i j}\right)=\frac{1}{1+e^{-\alpha\left(\frac{R_{i j}}{R_{0}}-1\right)}}
$$

where $R_{0}$ is the sum of atomic Van der Waals radii and $\alpha$ is a damping parameter. This function removes the singularity at $R_{i j}=0$ and ensures that the dispersion contribution becomes insignificant below the Van der Waals separation and, consequently, the covalent bonds described by the MM potential are not significantly affected.

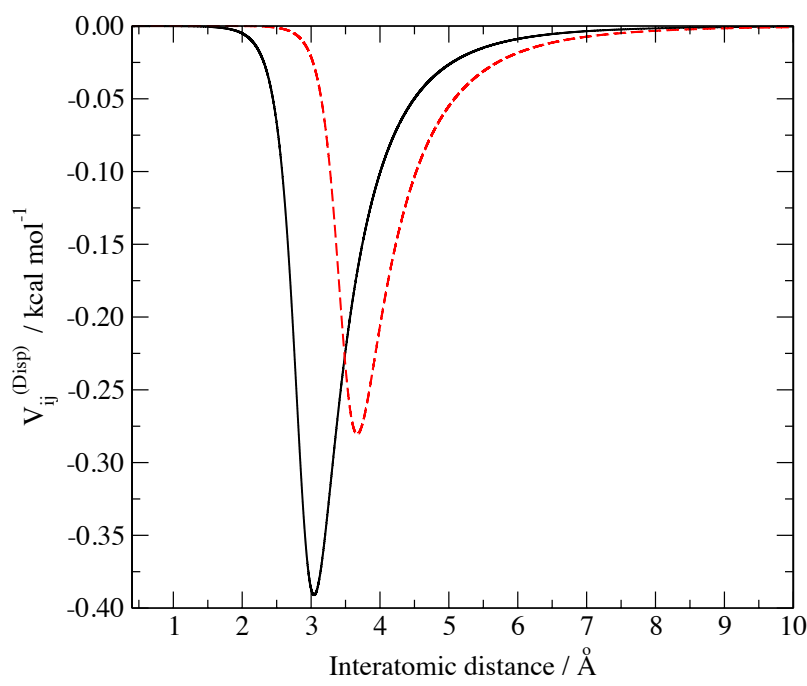

Figure 2: The variation of the Grimme dispersion correction with atomic distance for B3LYP (solid black line) and $\mathrm{MM}^{V i b+D}$ (broken red line) parameters.

The dispersion term has been optimized such that it predicts the interlayer spacing (3.35 $\AA$ ) and the frequency of the shear mode $\left(31 \mathrm{~cm}^{-1}\right)$ of bilayer graphene correctly. Using the existing parameters for the widely used B3LYP exchange-correlation functional gave values of $3.16 \AA$ and $11 \mathrm{~cm}^{-1}$ for the interlayer spacing and shear mode frequency, respectively. The value for $R_{0}$ of $1.452 \AA$ used for B3LYP is significantly lower than the sum of the experimental Van der Waals radii, and we use a value of $1.75 \AA$ which is more consistent with experiment. The value for $C_{6}^{i j}$ is not changed from the B3LYP parameterisation, and the values of $s_{6}$ and $\alpha$ have been modified such that the experimental values for bilayer graphene are predicted correctly. For systems that contain a single atom type, such as the ones considered here, the separate consideration of the $C_{6}^{i j}$ and $s_{6}$ parameters is not necessary but for systems with 
different atom types, separate consideration of these parameters might be required. The full parameters for the potential are given in Table 1 and the variation of the dispersion term with interatomic distance is shown in Figure 2. We note that the choice of the parameters for the dispersion term are not unique and several different parameterisations have been explored, but we find the parameters used give results consistent with known data for larger systems (see later).

Table 1: Parameters for the Murrell-Mottram potential with dispersion

\begin{tabular}{ll}
\hline Parameter & $\mathrm{MM}^{\mathrm{Vib}+D}$ \\
\hline$D / \mathrm{eV}$ & 6.298 \\
$r_{e} / \AA$ & 1.313 \\
$a_{2}$ & 7.428 \\
$a_{3}$ & 8.072 \\
$c_{0}$ & 7.788 \\
$c_{1}$ & 3.917 \\
$c_{2}$ & -17.503 \\
$c_{3}$ & -51.427 \\
$c_{4}$ & 99.263 \\
$c_{5}$ & -39.772 \\
$c_{6}$ & 70.505 \\
$c_{7}$ & 73.262 \\
$c_{8}$ & 3.831 \\
$c_{9}$ & 65.696 \\
$c_{10}$ & -85.307 \\
$s_{6}$ & 2.06 \\
$C_{6}^{i j} / \mathrm{J} \mathrm{nm}^{6} \mathrm{~mol}^{-1}$ & 1.75 \\
$R_{0} / \AA$ & 1.75 \\
$\alpha$ & 28.00 \\
\hline
\end{tabular}

In order to simulate Raman spectra it is necessary to compute the Raman intensities associated with the normal modes. This is achieved using the empirical bond polarisabilty model (BPM). ${ }^{61,62}$ In this approach the bond polarisability for a pair of atoms is given as

$$
\Pi_{\mu \nu}=\frac{1}{3}\left(\alpha_{\|}+2 \alpha_{\perp}\right) \delta_{\mu \nu}+\left(\alpha_{\|}-\alpha_{\perp}\right)\left(\frac{R_{\mu} R_{\nu}}{R^{2}}-\frac{1}{3} \delta_{\mu \nu}\right)
$$

where $\mu$ and $\nu$ are Cartesian coordinates and $\mathbf{R}$ is the vector connecting the two atoms linked by the bond. Raman intensities can be calculated from derivatives of equation 13 with re- 
spect to the normal modes, and equations for these derivatives can be found elsewhere. ${ }^{43,63}$ $\left(\alpha_{\|}+2 \alpha_{\perp}\right)$ and $\left(\alpha_{\|}-\alpha_{\perp}\right)$ and the associated derivatives $\left(\alpha_{\|}^{\prime}+2 \alpha_{\perp}^{\prime}\right),\left(\alpha_{\|}^{\prime}-\alpha_{\perp}^{\prime}\right)$ are empirical parameters. Here we adopt the parameters used by Saito et al., for modelling CNTs ${ }^{62}$ and graphene. ${ }^{61}$ For CNT $\left(\alpha_{\|}^{\prime}+2 \alpha_{\perp}^{\prime}\right)=4.7 \AA^{2},\left(\alpha_{\|}^{\prime}-\alpha_{\perp}^{\prime}\right)=4.0 \AA^{2}$ and $\left(\alpha_{\|}-\alpha_{\perp}\right)=0.04$ $\AA^{3}$ and for graphene $\left(\alpha_{\|}^{\prime}+2 \alpha_{\perp}^{\prime}\right)=7.55 \AA^{2},\left(\alpha_{\|}^{\prime}-\alpha_{\perp}^{\prime}\right)=2.60 \AA^{2}$ and $\left(\alpha_{\|}-\alpha_{\perp}\right)=0.32$ $\AA^{3}$. The BPM needs to be modified to account for the interlayer interactions. We use the approach of Luo et al., ${ }^{64}$ wherein Van der Waals interactions are treated as weak covalent bonds between layers, and that the BPM includes these bonds as well. With this treatment the Raman spectrum can be decomposed to purely Van der Waals interlayer modes or covalent vibrational mode contributions. The parameters used in the BPM have not be re-optimized for the interlayer modes since we are primarily concerned with identifying the Raman active modes rather than a quantitative prediction of the Raman intensities and the sparsity of experimental data makes such a parametrisation difficult.

Within the framework of this empirical model it is possible to perform calculations for very large systems containing thousands of atoms. Calculations can also be performed with periodic boundary conditions, opening the possibility to have large unit cells. Structures were optimized using the conjugate gradient method with a gradient convergence criterion of $10^{-8} E_{h} \AA^{-1}$, and a spherical cut-off was applied to the potential with a radius of $8 \AA$. The value for the cut-off was chosen such that the root mean squared error in the computed frequencies for model systems was less than $0.1 \mathrm{~cm}^{-1}$. Harmonic vibrational frequencies and normal modes were calculated through diagonalisation of the mass-weighted hessian matrix, and in this work the frequencies of the inter-layer vibrational modes are not scaled. The vibrational modes were visualized using IQMOL, and Raman spectra were generated by convolution with Lorentzian functions. 


\section{Results and Discussion}

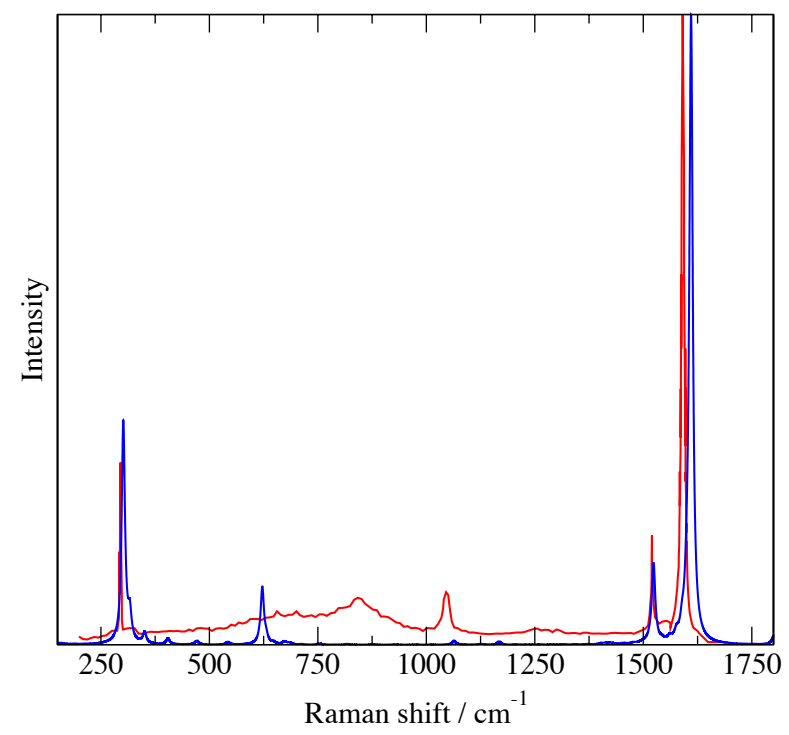

Figure 3: A comparison of a typical experimental Raman spectrum of a $(6,6)$ carbon nanotube $^{65}$ (red) with the simulated spectrum using $\mathrm{MM}^{V i b}$ and the BPM (blue). The computed spectrum generated using a $\mathrm{FWHM}=20 \mathrm{~cm}^{-1}$.

Figure 3 shows a comparison between Raman spectra for a $(6,6)$ single-walled nanotube computed with the $\mathrm{MM}^{\text {vib }}$ potential for a carbon nanotube of $8 \mathrm{~nm}$ length and experiment. The computed spectrum shows the $\mathrm{G}^{-}\left(1524 \mathrm{~cm}^{-1}\right)$ and $\mathrm{G}^{+}\left(1611 \mathrm{~cm}^{-1}\right)$ bands and the RBM $\left(300 \mathrm{~cm}^{-1}\right)$ in excellent agreement with experiment with respect to the frequency and relative intensity. The intermediate frequency band is also evident in the computed spectrum at 622 $\mathrm{cm}^{-1}$. The additional intensity observed in experiment in the mid-frequency range that is not present in the calculation is likely to be associated with the fact that the calculations consider a single pristine nanotube while the experiment will measure an imperfect nanotube bundle. This demonstrates that the $\mathrm{MM}^{\text {vib }}$ potential does provide a good description of the vibrational modes of single-layer $\mathrm{sp}^{2}$ based carbon materials.

FLG is the simplest and most extensively characterised multi-layered carbon material to assess the $\mathrm{MM}^{V i b+D}$ potential. Figure 4 shows the relationship of the calculated shear mode frequency for FLG with the number of graphene layers $N_{L}$ for up to 8 layers. The shearing mode reported in these calculations are the highest frequency Raman active modes and are 


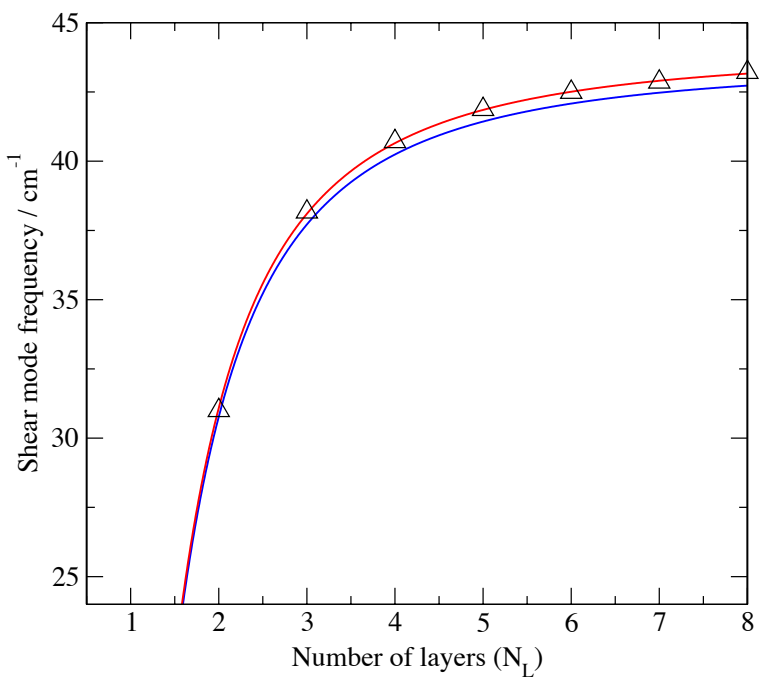

Figure 4: The variation in the shear mode frequency as a function of the number of layers, $N$, of graphene computed using $\mathrm{MM}^{\mathrm{Vib}+D}$ (triangles) and its fitting to equation 1 (red) and experimental fitting (blue). ${ }^{11}$

computed using periodic boundary conditions with a unit cell of dimensions $51.22 \times 51.75$ $\AA$ in the plane of graphene and the AB stacking pattern. The calculations reproduce the trend observed in experiment and predict the frequencies to within about a $1 \mathrm{~cm}^{-1}$ error. Fitting the theoretical data to equation 1 gives an interlayer coupling value of $13.1 \times 10^{18}$ $\mathrm{Nm}^{-1}$, which is in excellent agreement with the experimental value of $12.8 \times 10^{18} \mathrm{Nm}^{-1} .^{11}$ The calculations also showed the frequency of the $\mathrm{G}$ band to be independent of the number of layers.

FLG with $N_{L}$ layers will have $N_{L}-1$ shear modes. These additional shearing modes will have different displacement patterns and frequencies. These modes can be either Raman active, IR active or both, however, experiments have not yet been able to probe these modes in detail owing to their low intensities. Figure 5 shows the range of shearing modes and the computed frequencies for 2LG to 5LG and bulk graphite. Also included are the DFT-LDA computed frequencies from earlier studies. ${ }^{9,11}$ The use of LDA to study layered carbon materials is counter-intuitive since this exchange-correlation functional does not explicitly describe dispersion. However, LDA is widely used to study these materials and has been shown to give excellent results for the in-plane and $c$-axis lattice constants, as well 


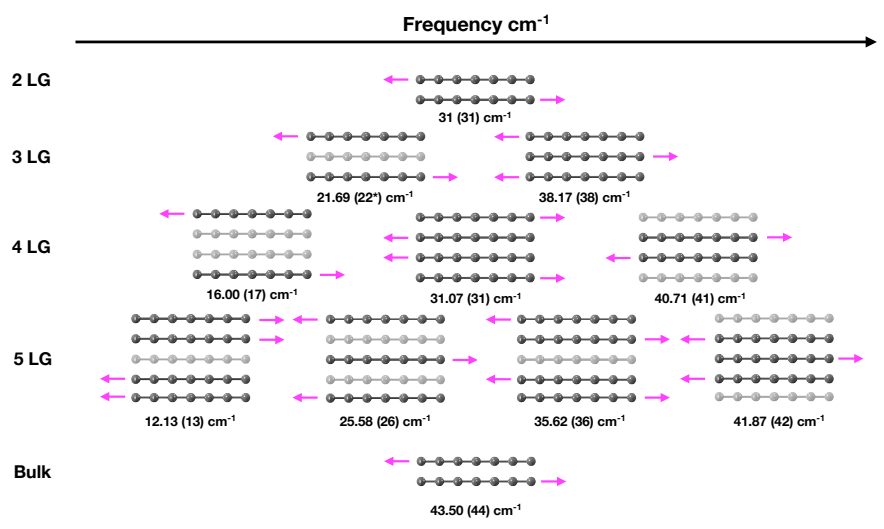

Figure 5: The normal mode displacements and frequencies for in-plane shear modes of NLG for $N=2-5$ and bulk graphite computed using $\mathrm{MM}^{V i b+D}$. Ab initio DFT-LDA frequencies from Ferrari et al. ${ }^{11}$ are given in the parenthesis. ${ }^{*}$ Value taken from Ping-Heng et al. ${ }^{9}$

as interlayer binding energies of graphite. ${ }^{66-70}$ This success is likely to be predicated on a fortuitous cancellation of errors. There is a very close agreement between the frequencies from the $\mathrm{MM}^{V i b+D}$ potential and DFT-LDA. We note that the method used here is much less computationally expensive than DFT, and calculations presented have a small fraction of the computational cost of comparable DFT-LDA calculations.

The computed Raman spectra for 3-layer graphene are shown in Figure 6. In these calculations the graphene layers lie in the $x y$-plane. The shearing modes appear in the spectrum with $x y$-polarisation since these vibrational modes comprise of in-plane displacements. Both of these modes are known to be Raman active. ${ }^{11}$ We note that the intensities are derived from the BPM using parameters that were developed for the intralayer vibrational modes. Consequently, the predicted intensities should be regarded a qualitative. In the $z$-polarised spectrum the inter-layer breathing mode is observed. The predicted frequency of the this mode is higher than the experimental value of $90 \mathrm{~cm}^{-1}$ derived from the overtone band. ${ }^{9}$

The consistency between the predictions of the $\mathrm{MM}^{V i b+D}$ potential and the established data for FLG provides a foundation to apply the potential to study some less well understood systems. Here we investigate a set of prototypical multi-walled armchair nanotubes: $\quad(10,10) @(15,15),(15,15) @(20,20),(20,20) @(25,25),(10,10) @(15,15) @(20,20)$ and $(10,10) @(15,15) @(20,20) @(25,25)$, where the notation $(10,10) @(15,15)$ denotes a nanotube 

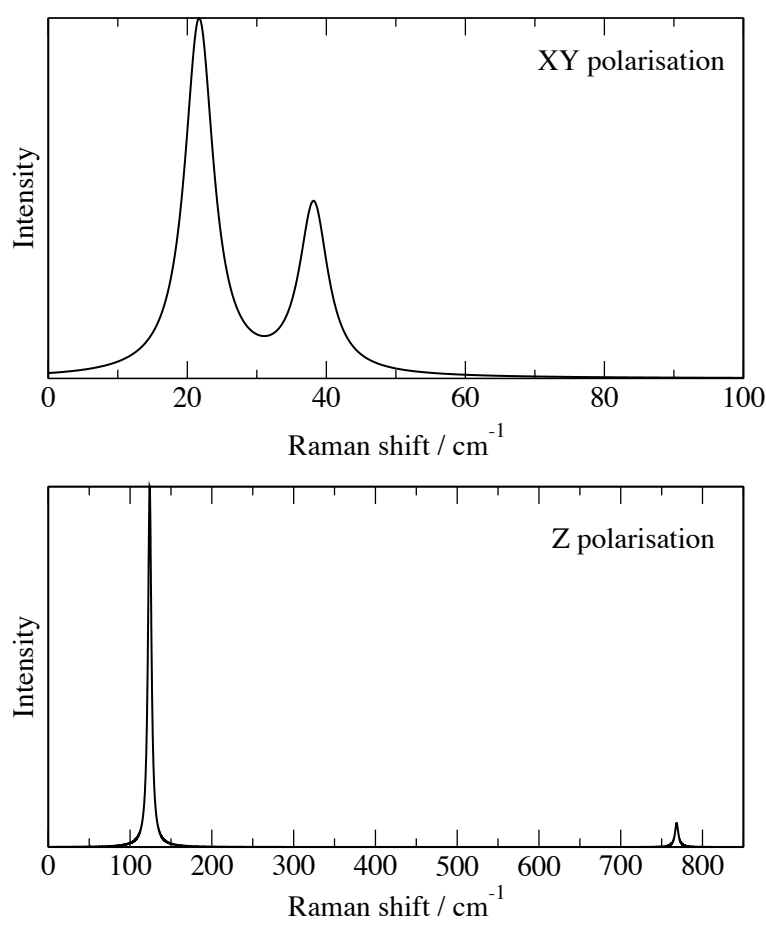

Figure 6: The calculated Raman spectra of 3-layer graphene with $x y$ and $z$-polarisation. The spectra are generated using a $\mathrm{FWHM}=5 \mathrm{~cm}^{-1}$.

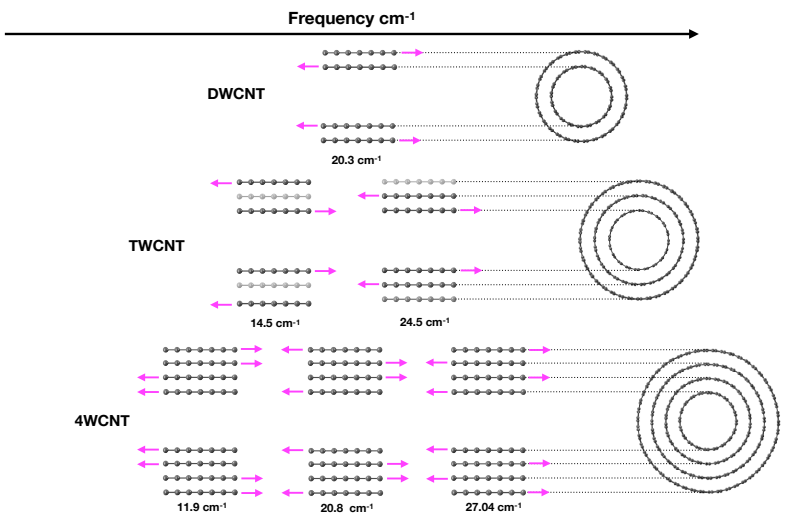

Figure 7: The normal mode displacements and frequencies for the linear shear modes of MWCNTs computed using MM $M^{V i b+D}$ for $(10,10) @(15,15),(10,10) @(15,15) @(20,20)$ and $(10,10) @(15,15) @(20,20) @(25,25)$. 
with chiral indices $(10,10)$ inside one with chiral indices $(15,15)$. The interlayer separation for these nanotubes lies between 3.37-3.45 $\AA$, consistent with experimentally observed tubes, ${ }^{71}$ and with a unit cell of length $48.2 \AA$ along the axis of the nanotube. Similar to FLG, for a given number of tubes, $N_{T}$, there will be $N_{T}-1$ shearing modes. In MWCNTs two types of shearing modes emerge, a set of linear modes that involve displacement along the tube axis and a set of rotational modes that involve displacement around the tube axis. These modes along with the calculated frequencies are shown in figures 7 and 8 . The calculations show the frequencies of the linear shearing modes to be larger, with an analogous progression to the modes seen in FLG. The same can be said for the rotational modes, but the frequencies are much lower for these modes. The lower frequencies of the rotational modes indicates a lower energy of rotation compared with linear displacement. Double-walled tubes have one rotational and one linear shear mode. Figure 9 shows the variation of the frequency of these modes with the diameter of the inner tube. The linear shear mode shows no strong dependence on the diameter and is approximately constant at $20.5 \mathrm{~cm}^{-1}$. In contrast, the rotational shear mode decreases significantly in frequency with increasing diameter, converging very close to zero.

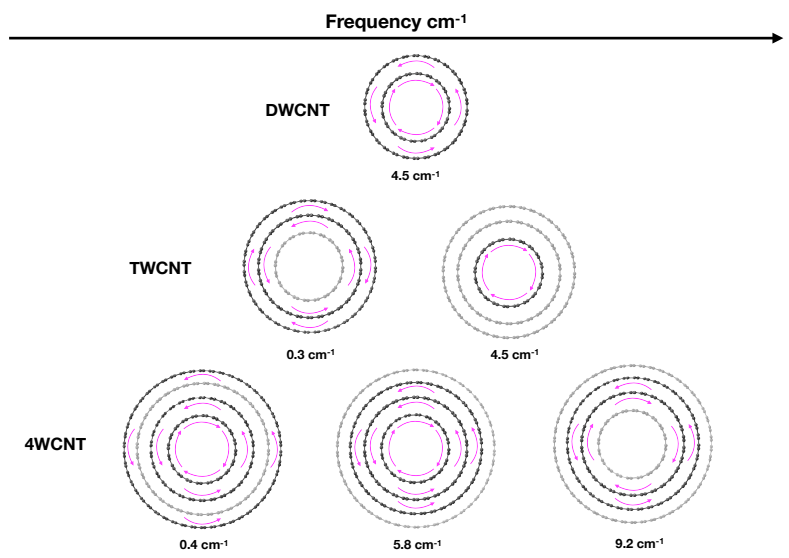

Figure 8: The normal mode displacements and frequencies for the rotational shearing modes of MWCNTs computed using $\mathrm{MM}^{V i b+D}$ for $(10,10) @(15,15),(10,10) @(15,15) @(20,20)$ and $(10,10) @(15,15) @(20,20) @(25,25)$.

Popov and Henrard employed a fixed force constant model with a Lennard-Jones po- 


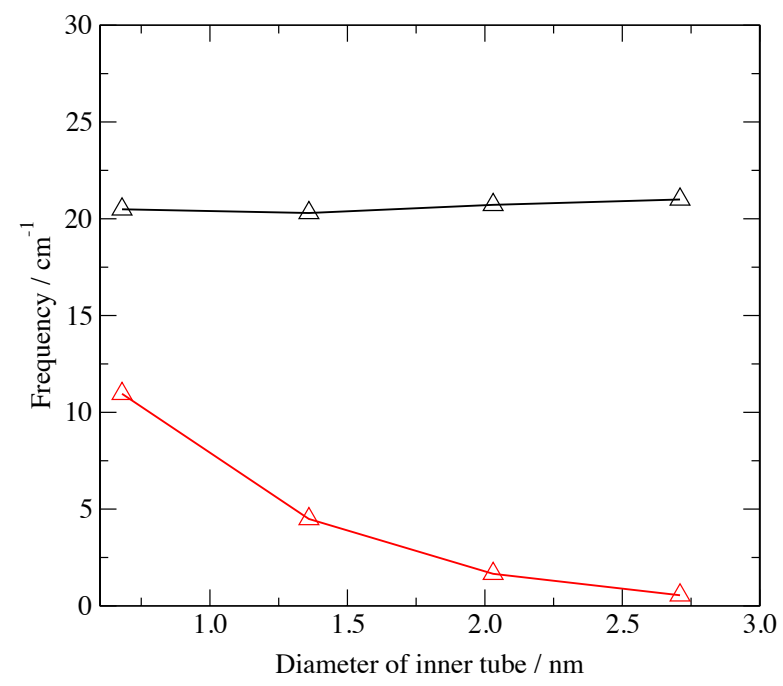

Figure 9: Variation of the frequencies of the rotational (red) and linear (black) shear modes with the diameter of the inner tube in a DWCNT. The dimensions of the tube at 0.68 (1.36), 1.36 (2.03), 2.03 (2.71) and 2.71 (3.39), where the outer tube diameter is in parenthesis.

tential between the concentric tubes to study the RBMs of MWCNTs. ${ }^{48}$ It was established that RBMs in MWCNTs with similar frequencies interact, leading to vibrational modes of strongly mixed character. These modes can be referred to as coupled radial breathing modes (CRBMs). Figure 10 shows the CRBMs computed using $\mathrm{MM}^{V i b+D}$ for a series of armchair MWCNTS with the corresponding SWCNT RBMs and contributing tubes to the normal mode shown with arrows. The data shows that as the number of walls in the tube increases, the degree of mixing of the RBMs tends to increase. In all cases the totally in-phase mode has the lowest frequency, while the out-of-phase mode has the highest frequency. The CRBMs in between comprise a mixture of in-phase and out of phase motions. This is physically intuitive since it suggests that the energy barrier for the in-phase motions is lower. It is also possible to compare the vibrational frequencies of the coupled modes with the frequencies of the respective isolated nanotubes. For the double-walled $(10,10) @(15,15)$ nanotube there is a significant shift in the frequencies of the RBMs, despite there only being a small degree of mixing of the modes. There is a shift to higher frequency of $16.9 \mathrm{~cm}^{-1}$ for the $(15,15)$ and an even larger shift of $38.7 \mathrm{~cm}^{-1}$ for the inner $(10,10)$ tube. For the triple-walled nanotube the low frequency mode associated with the outer tube is shifted by $19.4 \mathrm{~cm}^{-1}$, while the 
modes arising from the inner tubes are shifted by over $45 \mathrm{~cm}^{-1}$, in particular the $(15,15)$ tube is now restrained from the inner direction by the $(10,10)$ tube and from the outer by the $(20,20)$ tube. There is also a further increase in the highest frequency mode, and this trend continues for the four nanotube system. When there is a large difference in RBM frequencies, for example in the $(5,5) @(10,10)$ double-walled nanotube, there is no, or very little, coupling between the RBMs. The computed Raman spectra for the low frequency region of the MWCNTs are shown in figure 11. The spectra in this region are dominated by the CRBMs and the shearing modes are too weak to feature. The calculations predict that the most intense mode is one with the lowest frequency and corresponds to the in-phase CRBM.

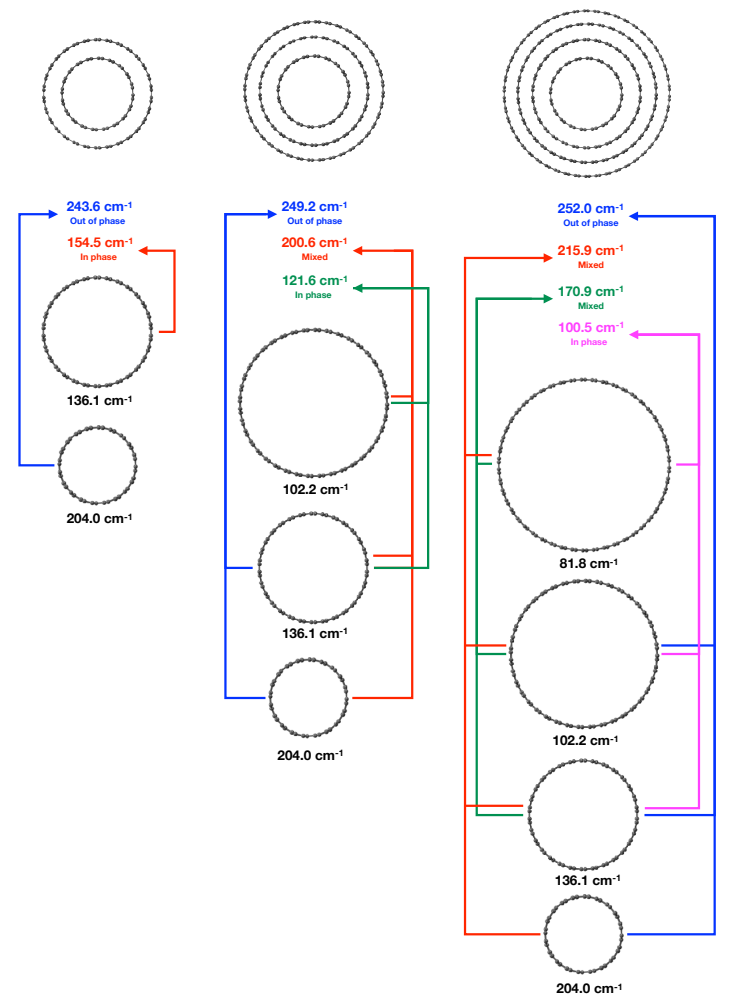

Figure 10: RBMs and CRBMs of the SWNTs $(10,10), \quad(15,15)$ and $(20,20)$ and their MWCNT counterparts $(10,10) @(15,15), \quad(10,10) @(15,15) @(20,20)$ and $(10,10) @(15,15) @(20,20) @(25,25)$ with the contributing SWNTs to the CRBMs shown with arrows.

A more recent class of carbon nanomaterials are graphitic nanofibres which comprise 


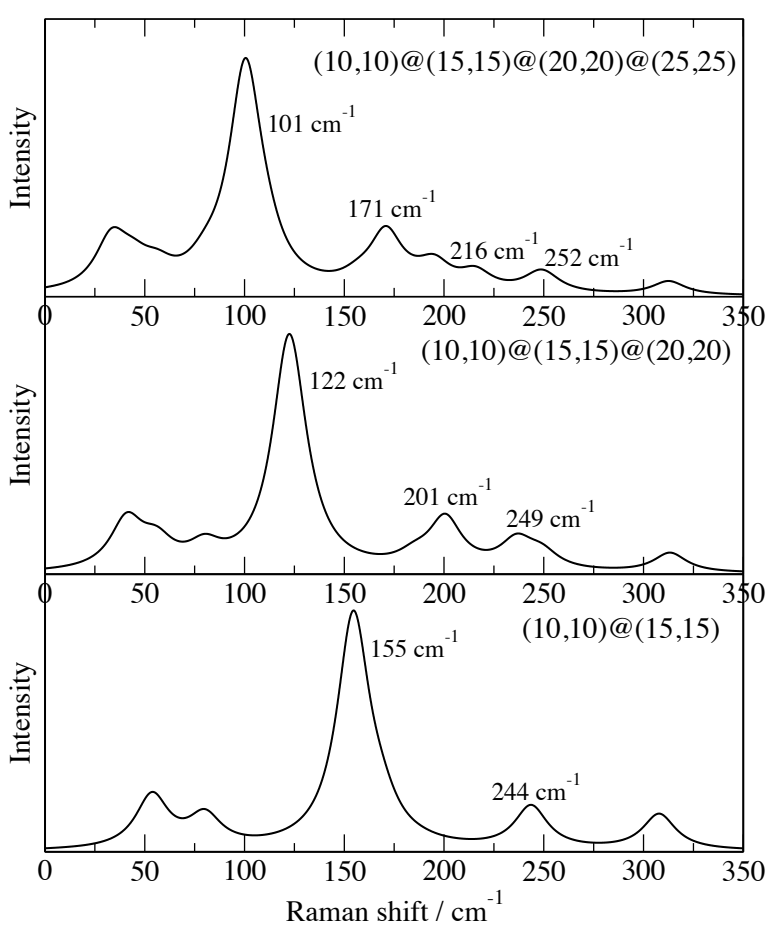

Figure 11: Computed Raman spectra for the MWCNTs with the frequencies of the CRBMs shown.

layers of nanocones stacked parallel or at an angle from the fibre axis. Nanofibres have been observed in transmission electron microscopy and can have either a solid or hollow core. These structures have a large number of reactive edges making them versatile materials. ${ }^{72,73}$ Here we consider hat stacked carbon nanofibres which are the multi-layer analogues of nanocones. These materials have a separation of $0.34 \mathrm{~nm}$, which is comparable to FLG. The attraction of these materials arise from applications in polymer conductive composites which are materials that exhibit superior electrical and thermal properties compared to conventional conductive polymer composites. ${ }^{74}$ The Raman spectroscopy of nanocones and the associated nanofibres is not well characterised, and here we explore the relationship between structure and Raman spectra for these materials.

Before considering nanofibres, we will consider the Raman spectroscopy of nanocones. Carbon nanocones are conical carbon nanostructures, which can be viewed as curved graphite sheets formed as open cones. These cones are constructed by removing sections of graphene 


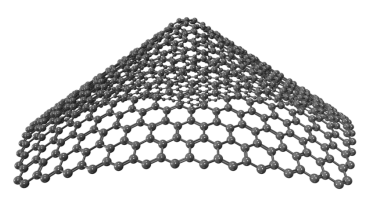

(a) $n=1, \chi=112.9^{\circ}$

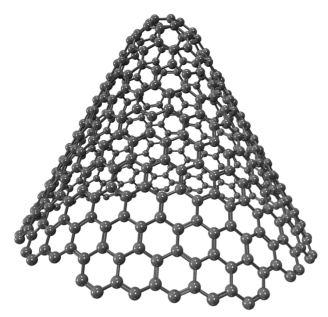

(c) $n=3, \chi=60.0^{\circ}$

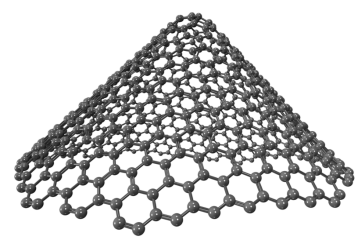

(b) $n=2, \chi=83.6^{\circ}$

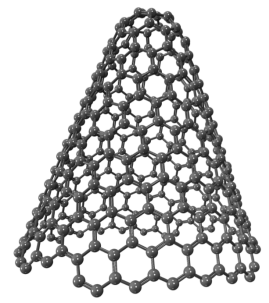

(d) $n=4, \chi=38.9^{\circ}$

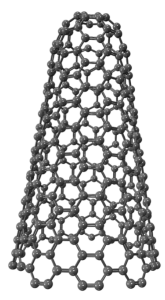

(e) $n=5, \chi=19.2^{\circ}$

Figure 12: Carbon nanocone structures with apex pentagon number, $n$ and apex angle, $\chi$.

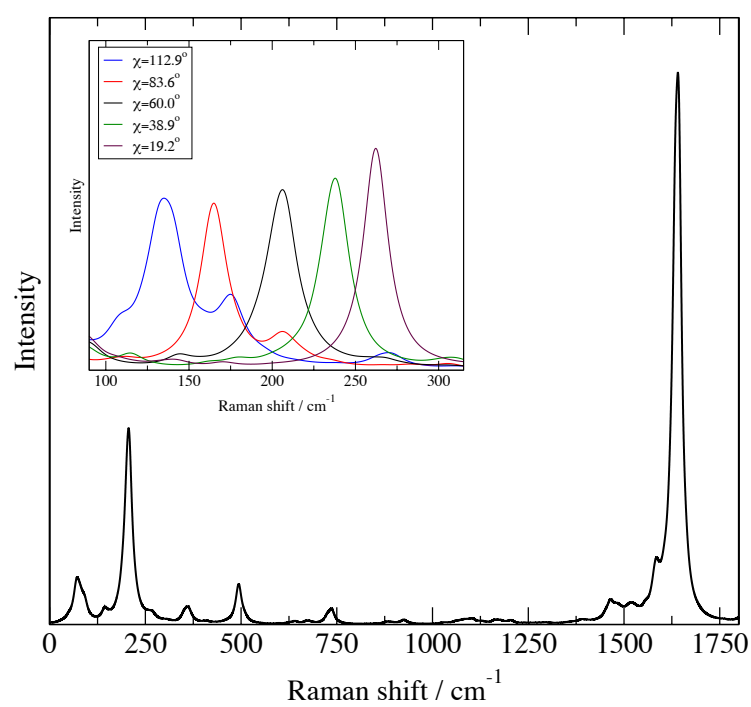

Figure 13: Computed Raman spectra for the carbon nanocone $\chi=60^{\circ}$. Inset the CBMs for $\chi=19.2-112.9^{\circ}$. 
at $n \times 60^{\circ}(n=1-5)$ intervals and connecting the resultant edges (shown in figure 12). The strain at the cone apex results in $n$ pentagons with apex angles $(\chi)$ of $112.9^{\circ}, 83.6^{\circ}$, $60.0^{\circ}, 38.9^{\circ}$ and $19.2^{\circ}{ }^{75}$ There are currently few studies into the Raman spectroscopy of these systems, however, a study by Campos et al. ${ }^{76}$ utilised the shift in G band of these systems to map out the strain over a nanocone through Raman imaging. Figure 13, shows the computed Raman spectra for the carbon nanocone with $\chi=60^{\circ}$. The highest intensity mode lies at $1639 \mathrm{~cm}^{-1}$ and corresponds to the $\mathrm{G}$ band for these materials. In the lower frequency region $\left(0-400 \mathrm{~cm}^{-1}\right)$ region lies the conical breathing mode $(\mathrm{CBM})$. The spectra show that the frequency of this mode increases with the apex angle, and this increase is linear in the region studied and fits the equation $\omega_{C B M}\left(\mathrm{~cm}^{-1}\right)=-1.41 \chi+289.54$. Such a relationship could facilitate the assignment of carbon nanocones in experiment and determination of distributions. The frequency of the $\mathrm{G}$ band also depends on the apex angle, with it varying between $1613 \mathrm{~cm}^{-1}$ for $\chi=19.2^{\circ}$ and quickly converging to $1648 \mathrm{~cm}^{-1}$ for $\chi=19.2^{\circ}$. This is not surprising since $\mathrm{MM}^{V i b}$ predicts a $\mathrm{G}$ band frequency of $1610 \mathrm{~cm}^{-1}$ for a typical nanotube and small angle cones will have structures closer to a cylinder, while those with wider angles tend to have much flatter structure have a value closer graphene.

In order to explore the potential of Raman spectroscopy as a tool for characterising the structure of nanofibres we study a nanofibre constructed from a $n=3, \chi=60.0^{\circ}$ nanocone with either 3 or 4 units. As discussed previously, these nanofibres can have either a solid or hollow core. The hollow core is elliptical in shape and its size is defined as $d_{t}$, where $d_{t}$ is the length of the major axis of the ellipse. These structures are illustrated in figure 14. The simulation of the Raman spectra for the hollow core structures reveal two Raman active modes in the low frequency region. These two modes have the characteristics of a bending mode and RBM, and are referred to as a distorted bending mode (d-BM) and distorted radial breathing mode $(\mathrm{d}-\mathrm{RBM})$, respectively. The frequency of the both of these modes increases with increasing diameter of the cone. This is in contrast to the well established behaviour of the RBM in CNTs and is a consequence of the the mass decreasing with increasing diameter. 


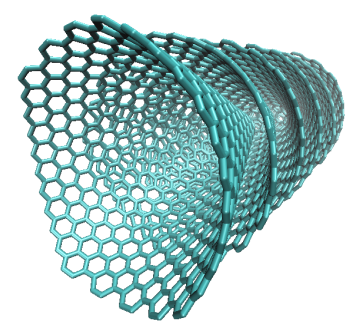

(a)
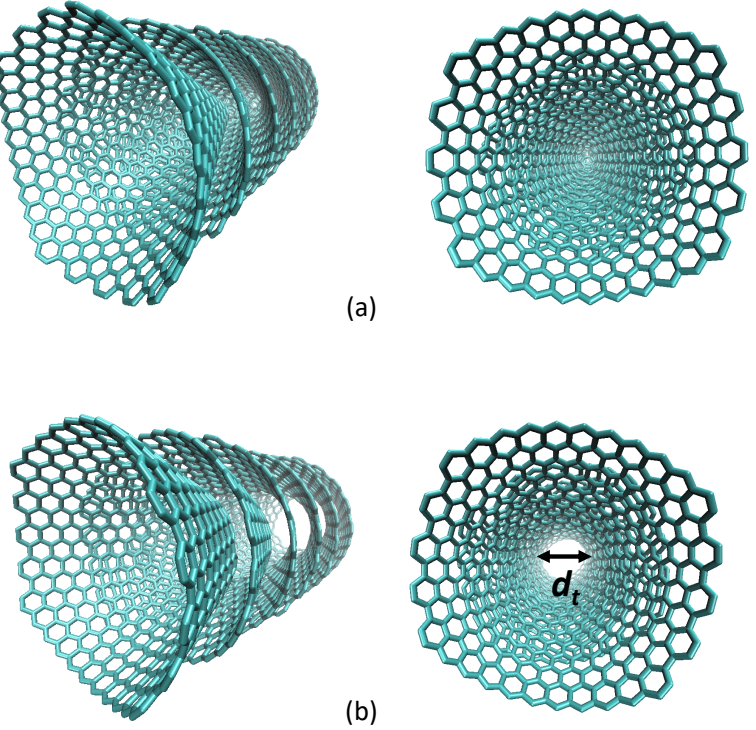

Figure 14: Representation of the structure of a solid (a) and hollow (b) cup stacked graphitic nanofiber built from a $n=4, \chi=60.0^{\circ}$.

\section{Conclusions}

An empirical potential for carbon that has been designed for the calculation of the vibrational frequencies of carbon nanomaterials has been extended to include a dispersion term. This term has been parameterised to predict the inter-layer spacing and shear mode frequency of bi-layer graphene correctly. When combined with the BPM for Raman intensities it provides a computationally inexpensive approach for the simulation of the Raman spectroscopy of layered carbon nanomaterials. This approach can accurately describe the variation in the shearing mode frequencies of FLG, and has been applied to study the Raman active low frequency modes in MWCNTs and a carbon based nanofibre. The RBMs in MWCNTs can couple together, and the shift to higher frequency compared with the isolated nanotubes can be quantified. The resulting mode with the lowest frequency corresponds to an in-phase vibration and is predicted to have the greatest intensity. The low frequency Raman active 
modes identified for the nanofibre are sensitive to the core diameter of the fibre and have the potential to be used to characterise these materials. This approach provides a platform for investigating the effect of structural defects on the low frequency modes, and these studies are currently underway.

\section{Acknowledgements}

The authors would like to thank the University of Nottingham for access to its High Performance Computing facility and the award of a studentship to PMT.

\section{Conflicts of Interest}

There are no conflicts to declare.

\section{References}

(1) Iijima, S. Helical microtubules of graphitic carbon. Nature 1991, 354, 56-58.

(2) Novoselov, K. S.; Geim, A. K.; Morozov, S. V.; Jiang, D.; Zhang, Y.; Dubonos, S. V.; Grigorieva, I. V.; Firsov, A. A. Electric field effect in atomically thin carbon films. Science 2004, 306, 666-669.

(3) Baughman, R. H.; Zakhidov, A. A.; de Heer, W. A. Carbon nanotubes-the route toward applications. Science 2002, 297, 787-792.

(4) De Volder, M. F. L.; Tawfick, S. H.; Baughman, R. H.; Hart, A. J. Carbon nanotubes: Present and future commercial applications. Science 2013, 339, 535-539.

(5) Dresselhaus, M. S.; Dresselhaus, G.; Saito, R.; Jorio, A. Raman spectroscopy of carbon nanotubes. Phys. Rep. 2005, 409, 47. 
(6) Malard, L. M.; Pimenta, M. A.; Dresselhaus, G.; Dresselhaus, M. S. Raman spectroscopy in graphene. Phys. Rep. 2009, 473, 51-87.

(7) Jorio, A.; Pimenta, M. A.; Filho, A. G. S.; Saito, R.; Dresselhaus, G.; Dresselhaus, M. S. Characterizing carbon nanotube samples with resonance Raman scattering. New J. Phys. 2003, 5, 139.

(8) Graupner, R. Raman spectroscopy of covalently functionalized single-wall carbon nanotubes. J. Raman Spectrosc. 2007, 38, 673-683.

(9) Lin, M. L.; Wu, J. B.; Liu, X. L.; Tan, P. H. Probing the shear and layer breathing modes in multilayer graphene by Raman spectroscopy. J. Raman Spectrosc. 2018, 49, $19-30$.

(10) Wu, J.-B.; Lin, M.-L.; Cong, X.; Liu, H.-N.; Tan, P.-H. Raman spectroscopy of graphene-based materials and its applications in related devices. Chem. Soc. Rev. 2018, $47,1822-1873$.

(11) Tan, P. H.; Han, W. P.; Zhao, W. J.; Wu, Z. H.; Chang, K.; Wang, H.; Wang, Y. F.; Bonini, N.; Marzari, N.; Pugno, N.; Savini, G.; Lombardo, A.; Ferrari, A. C. The shear mode of multilayer graphene. Nature Materials 2012, 11, 294.

(12) Lui, C. H.; Ye, Z.; Keiser, C.; Barros, E. B.; He, R. Stacking-dependent shear modes in trilayer graphene. Appl. Phys. Lett. 2015, 106, 041904.

(13) Lui, C. H.; Heinz, T. F. Measurement of layer breathing mode vibrations in few-layer graphene. Physical Review B 2013, 87, 121404.

(14) Lefrant, S. Raman and SERS studies of carbon nanotube systems. Curr. Appl. Phys. 2002, 2, 479-482.

(15) Benoit, J. M.; Buisson, J. P.; Chauvet, O.; Godon, C.; Lefrant, S. Low-frequency Raman 
studies of multiwalled carbon nanotubes: Experiments and theory. Phys. Rev. B 2002, 66, 073417.

(16) Zhao, X.; Ando, Y.; Qin, L.-C.; Kataura, H.; Maniwa, Y.; Saito, R. Characteristic Raman spectra of multiwalled carbon nanotubes. Physica B: Condensed Matter 2002, 323, 265-266.

(17) Basirjafari, S.; Esmaielzadeh Khadem, S.; Malekfar, R. Determination of the inner diameter of a double-walled carbon nanotube from its Raman spectra. J. Appl. Phys. 2013, 113, 064304.

(18) Lehman, J. H.; Terrones, M.; Mansfield, E.; Hurst, K. E.; Meunier, V. Evaluating the characteristics of multiwall carbon nanotubes. Carbon 2011, 49, $2581-2602$.

(19) Rebelo, S. L. H.; Guedes, A.; Szefczyk, M. E.; Pereira, A. M.; Araújo, J. P.; Freire, C. Progress in the Raman spectra analysis of covalently functionalized multiwalled carbon nanotubes: unraveling disorder in graphitic materials. Phys. Chem. Chem. Phys. 2016, 18, 12784-12796.

(20) Choi, C. H.; Kertesz, M.; Mihaly, L. Vibrational assignment of all 46 fundamentals of $\mathrm{C}_{60}$ and $\mathrm{C}_{60}^{6-}$ : A scaled quantum mechanical results performed in redundant internal coordinates and compared to experiments. J. Phys. Chem. A 2000, 104, 102-112.

(21) Schettino, V.; Pagliai, M.; Ciabini, L.; Cardini, G. The vibrational spectrum of fullerene $\mathrm{C}_{60}$. J. Phys. Chem. A 2001, 105, 11192-11196.

(22) Schettino, V.; Pagliai, M.; Cardini, G. The infrared and Raman spectra of fullerene $\mathrm{C}_{70}$. DFT calculations and correlation with $\mathrm{C}_{60}$. J. Phys. Chem. A 2002, 106, 1815-1823.

(23) Sun, G.; Kertesz, M. Vibrational Raman spectra of $\mathrm{C}_{70}$ and $\mathrm{C}_{60}{ }^{6-}$ studied by density functional theory. J. Phys. Chem. A 2002, 106, 6381-6386. 
(24) Do, H.; Besley, N. A. Calculation of the vibrational frequencies of carbon clusters and fullerenes with empirical potentials. Phys. Chem. Chem. Phys. 2015, 17, 3898-3908.

(25) Adjizian, J.-J.; Vlandas, A.; Rio, J.; Charlier, J.-C.; Ewels, C. P. Ab initio infrared vibrational modes for neutral and charged small fullerenes $\left(\mathrm{C}_{20}, \mathrm{C}_{24}, \mathrm{C}_{26}, \mathrm{C}_{28}, \mathrm{C}_{30}\right.$ and C60). Philos. Trans. Royal Soc. A 2016, 374, 20150323.

(26) Chen, H.; Golder, M. R.; Wang, F.; Jasti, R.; Swan, A. K. Raman spectroscopy of carbon nanohoops. Carbon 2014, 67, $203-213$.

(27) Chen, H.; Golder, M. R.; Wang, F.; Doorn, S. K.; Jasti, R.; Tretiak, S.; Swan, A. K. Raman-active modes of even-numbered cycloparaphenylenes: Comparisons between experiments and density functional theory (DFT) Calculations with group theory arguments. J. Phys. Chem. C 2015, 119, 2879-2887.

(28) López-Chávez, E.; Cruz-Torres, A.; de Landa Castillo-Alvarado, F.; Ortíz-López, J.; Peña-Castañeda, Y. A.; Martínez-Magadán, J. M. Vibrational analysis and thermodynamic properties of $\mathrm{C}_{120}$ nanotorus: a DFT study. J. Nanoparticle Res. 2011, 13, 6649-6659.

(29) Besley, N. A.; Titman, J. J.; Wright, M. D. Theoretical Study of the 13C NMR Spectroscopy of Single-Walled Carbon Nanotubes. J. Am. Chem. Soc. 2005, 127, 1794817953.

(30) Besley, N. A.; Noble, A. NMR chemical shifts of molecules encapsulated in single walled carbon nanotubes. J. Chem. Phys. 2008, 128, 101102.

(31) Aydin, M.; Akins, D. L. Calculated dependence of vibrational band frequencies of single-walled and double-walled carbon nanotubes on diameter. Vib. Spectrosc. 2010, $53,163-172$. 
(32) Kupka, T.; Stachów, M.; Chełmecka, E.; Pasterny, K.; Stobiński, L. DFT calculations of structures, ${ }^{13} \mathrm{C}$ NMR chemical shifts and Raman RBM mode of simple models of ultra small diameter $(4,0)$ zigzag hydroxylated single wall carbon nanotubes. Synth. Met. 2012, 162, $573-583$.

(33) Yumura, T.; Nozaki, D.; Bandow, S.; Yoshizawa, K.; Iijima, S. End-Cap effects on vibrational structures of finite-length carbon nanotubes. J. Am. Chem. Soc. 2005, 12\%, 11769-11776.

(34) Kupka, T.; Stachow, M.; Stobinski, L.; Kaminsky, J. Calculation of Raman parameters of real-size zigzag (n, 0) single-walled carbon nanotubes using finite-size models. Phys. Chem. Chem. Phys. 2016, 18, 25058-25069.

(35) Saidi, W. A. Effects of topological defects and diatom vacancies on characteristic vibration modes and Raman intensities of zigzag single-walled carbon nanotubes. J. Phys. Chem. A 2014, 118, 7235-7241.

(36) Kahn, D.; Lu, J. P. Vibrational modes of carbon nanotubes and nanoropes. Phys. Rev. B 1999, 60, 6535-6540.

(37) Witek, H. A.; Irle, S.; Zheng, G.; de Jong, W. A.; Morokuma, K. Modeling carbon nanostructures with the self-consistent charge density-functional tight-binding method: Vibrational spectra and electronic structure of $\mathrm{C}_{28}, \mathrm{C}_{60}$, and $\mathrm{C}_{70}$. J. Chem. Phys. 2006, 125.

(38) Popov, V. N.; Henrard, L.; Lambin, P. Electron-phonon and electron-photon interactions and resonant Raman scattering from the radial-breathing mode of single-walled carbon nanotubes. Phys. Rev. B 2005, 72, 035436.

(39) Popov, V. N.; Lambin, P. Theoretical resonant Raman spectra of nanotube $(7,0)$ with point defects. Physica Status Solidi (b) 2009, 246, 2602-2605. 
(40) Jain, S. K.; Barkema, G. T.; Mousseau, N.; Fang, C.-M.; van Huis, M. A. Strong longrange relaxations of structural defects in graphene simulated using a new semiempirical potential. J. Phys. Chem. C 2015, 119, 9646-9655.

(41) Pool, A. J.; Jain, S. K.; Barkema, G. T. Structural characterization of carbon nanotubes via the vibrational density of states. Carbon 2017, 118, $58-65$.

(42) Tailor, P. M.; Wheatley, R. J.; Besley, N. A. An empirical force field for the simulation of the vibrational spectroscopy of carbon nanomaterials. Carbon 2017, 113, 299 - 308 .

(43) Guha, S.; Menéndez, J.; Page, J.; Adams, G. Empirical bond polarizability model for fullerenes. Phys. Rev. B 1996, 53, 13106-13114.

(44) Jiang, J.-W.; Tang, H.; Wang, B.-S.; Su, Z.-B. Raman and infrared properties and layer dependence of the phonon dispersions in multilayered graphene. Phys. Rev. B 2008, r7, 235421.

(45) Popov, V. N.; Van Alsenoy, C. Low-frequency phonons of few-layer graphene within a tight-binding model. Phys. Rev. B 2014, 90, 245429.

(46) Wang, X. Y.; Xu, C. L.; Wang, X. Radial breathing vibration of multiwall carbon nanotubes based on a rigorous van der Waals interaction. Model. Simul. Mater. Sci. Eng. 2006, 14, 759 .

(47) Sbai, K.; Rahmani, A.; Fakrach, B.; Chadli, H.; Benhamou, M. Modeling and simulation of vibrational breathing-like modes in individual multiwalled carbon nanotubes. Physica E 2014, 56, $312-318$.

(48) Popov, V. N.; Henrard, L. Breathinglike phonon modes of multiwalled carbon nanotubes. Phys. Rev. B 2002, 65, 235415.

(49) Grimme, S. Accurate description of van der Waals complexes by density functional theory including empirical corrections. J. Comp. Chem. 2004, 25, 1463-1473. 
(50) Murrell, J. N.; Mottram, R. E. Potential energy functions for atomic solids. Mol. Phys. 1990, 69, 571-585.

(51) Johnston, R. L.; Fang, J.-Y. An empirical many body potential energy function for aluminum. Application to solid phases and microclusters. J. Chem. Phys. 1992, 97, $7809-7821$.

(52) Fang, J.-Y.; Johnston, R. L.; Murrell, J. N. Potential-energy functions for Cu, Ag and Au solids and their application to clusters of these elements. J. Chem. Soc., Faraday Trans. 1993, 89, 1659-1665.

(53) Gao, F.; Johnston, R. L.; Murrell, J. N. Empirical many-body potential energy functions for iron. J. Phys. Chem. 1993, 97, 12073-12082.

(54) Besley, N. A.; Johnston, R. L.; Stace, A. J.; Uppenbrink, J. Theoretical study of the structures and stabilities of iron clusters. J. Mol. Struct. 1995, 341, $75-90$.

(55) Hearn, J. E.; Johnston, R. L.; Leoni, S.; Murrell, J. N. Global potentials for calcium and strontium solids. J. Chem. Soc., Faraday Trans. 1996, 92, 425-432.

(56) Lloyd, L. D.; Johnston, R. L. Modelling aluminium clusters with an empirical manybody potential. Chem. Phys. 1998, 236, $107-121$.

(57) Cox, H.; Johnston, R. L.; Ward, A. An empirical many-body potential energy function for modelling ytterbium. J. Phys. Condens. Matter 1998, 10, 9419.

(58) Cox, H.; Johnston, R. L.; Murrell, J. N. Empirical Potentials for Modeling Solids, Surfaces, and Clusters. J. Solid State Chem. 1999, 145, 517-540.

(59) Al-Derzi, A. R.; Johnston, R. L.; Murrell, J. N.; Rodriguez-Ruiz, J. A. Potential energy functions for atomic solids. Mol. Phys. 1991, 73, 265-282.

(60) Eggen, B. R.; Johnston, R. L.; Murrell, J. N. Carbon cluster structures and stabilities predicted from solid-state potentials. J. Chem. Soc., Faraday Trans. 1994, 90, 3029. 
(61) Saito, R.; Furukawa, M.; Dresselhaus, G.; Dresselhaus, M. S. Raman spectra of graphene ribbons. J. Phys. Condens. Matter 2010, 22, 334203.

(62) Saito, R.; Takeya, T.; Kimura, T.; Dresselhaus, G.; Dresselhaus, M. S. Raman intensity of single-wall carbon nanotubes. Phys. Rev. B 1998, 57, 4145-4153.

(63) Rahmani, A.; Sauvajol, J.-L.; Rols, S.; Benoit, C. Nonresonant Raman spectrum in infinite and finite single-wall carbon nanotubes. Phys. Rev. B 2002, 66, 125404.

(64) Luo, X.; Lu, X.; Cong, C.; Yu, T.; Xiong, Q.; Ying Quek, S. Stacking sequence determines Raman intensities of observed interlayer shear modes in 2D layered materials A general bond polarizability model. Sci. Rep. 2015, 5, 14565.

(65) Sanchez-Valencia, J. R.; Dienel, T.; Gröning, O.; Shorubalko, I.; Mueller, A.; Jansen, M.; Amsharov, K.; Ruffieux, P.; Fasel, R. Controlled synthesis of single-chirality carbon nanotubes. Nature 2014, 512, 61-64.

(66) Yin, M. T.; Cohen, M. L. Structural theory of graphite and graphitic silicon. Phys. Rev. B 1984, 29, 6996-6998.

(67) Jansen, H. J. F.; Freeman, A. J. Structural and electronic properties of graphite via an all-electron total-energy local-density approach. Phys. Rev. B 1987, 35, 8207-8214.

(68) Trickey, S. B.; Müller-Plathe, F.; Diercksen, G. H. F.; Boettger, J. C. Interplanar binding and lattice relaxation in a graphite dilayer. Phys. Rev. B 1992, 45, 4460-4468.

(69) Schabel, M. C.; Martins, J. L. Energetics of interplanar binding in graphite. Phys. Rev. $B$ 1992, 46, 7185-7188.

(70) Charlier, J.-C.; Gonze, X.; Michenaud, J.-P. Graphite Interplanar Bonding: Electronic Delocalization and van der Waals Interaction. Europhys. Lett. 1994, 28, 403.

(71) Kiang, C. H.; Endo, M.; Ajayan, P. M.; Dresselhaus, G.; Dresselhaus, M. S. Size effects in carbon nanotubes. Phys. Rev. Lett. 1998, 81, 1869-1872. 
(72) Endo, M.; Kim, Y. A.; Hayashi, T.; Fukai, Y.; Oshida, K.; Terrones, M.; Yanagisawa, T.; Higaki, S.; Dresselhaus, M. S. Structural characterization of cup-stacked-type nanofibers with an entirely hollow core. Appl. Phys. Lett. 2002, 80, 1267-1269.

(73) Sato, Y.; Shibata, K.-I.; Kataoka, H.; Ogino, S.-I.; Bunshi, F.; Yokoyama, A.; Tamura, K.; Akasaka, T.; Uo, M.; Motomiya, K.; Jeyadevan, B.; Hatakeyama, R.; Watari, F.; Tohji, K. Strict preparation and evaluation of water-soluble hat-stacked carbon nanofibers for biomedical application and their high biocompatibility: influence of nanofiber-surface functional groups on cytotoxicity. Mol. BioSyst. 2005, 1, 142-145.

(74) Al-Saleh, M. H.; Sundararaj, U. A review of vapor grown carbon nanofiber/polymer conductive composites. Carbon 2009, 47, 2-22.

(75) Naess, S. N.; Elgsaeter, A.; Helgesen, G.; Knudsen, K. D. Carbon nanocones: wall structure and morphology. Sci. Technol. Adv. Mater. 2009, 10, 065002.

(76) Campos, J. L. E.; Miranda, H.; Rabelo, C.; Sandoz-Rosado, E.; Pandey, S.; Riikonen, J.; Cano-Marquez, A.; Jorio, A. Applications of Raman spectroscopy in graphene-related materials and the development of parameterized PCA for large-scale data analysis. $J$. Raman Spectrosc. 2017, 49, 54-65. 